\title{
The Effect of Olanzapine on Craving and Alcohol Consumption
}

\author{
Kent E Hutchison*,', Lara Ray', Erica Sandman', Marie-Christine Rutter', Annie Peters', Dena Davidson ${ }^{2}$ \\ and Robert Swift ${ }^{3}$ \\ 'Department of Psychology, University of Colorado at Boulder, Boulder, CO, USA; ${ }^{2}$ Institute of Psychiatric Research, Indiana University - Purdue \\ University of Indianapolis, Indianapolis, IN, USA; ${ }^{3}$ Providence Veteran Affairs Medical Center and Center for Alcohol and Addiction Studies at \\ Brown University, Providence, RI, USA
}

\begin{abstract}
Previous studies have indicated that olanzapine decreases craving after a priming dose of alcohol, that craving after a priming dose of alcohol is greater among individuals with the seven-repeat allele of the DRD4 variable number of tandem repeats (VNTR) polymorphism, and that the effect of olanzapine (a D2/D4 antagonist) is more pronounced among individuals with this allele. The present study tested the hypothesis that olanzapine may be differentially effective at reducing cue-elicited craving and differentially effective as a treatment for alcohol dependence over the course of a 12-week, randomized, placebo-controlled trial among individuals with and without the seven-repeat allele. Participants who met DSM IV criteria for alcohol dependence were randomly assigned to receive olanzapine $(5 \mathrm{mg})$ or a placebo over the course of the trial. After 2 weeks of treatment, participants completed a cue reactivity assessment. The results suggested that participants who were homozygous or heterozygous for the seven (or longer)-repeat allele of the DRD4 VNTR responded to olanzapine with reductions in cue-elicited craving as well as reductions in alcohol consumption over the course of the 12-week trial, whereas individuals with the shorter alleles did not respond favorably to olanzapine.

Neuropsychopharmacology (2006) 31, |310-1317. doi:10.1038/sj.npp. 1300917; published online 12 October 2005
\end{abstract}

Keywords: alcohol; craving; olanzapine; gene; DRD4

\section{INTRODUCTION}

Previous studies have suggested that currently accepted pharmacotherapies (ie naltrexone, disulfram, and acamprosate) as well as medications currently under development (eg ondansetron, topiramate, and aripiprazole) for alcohol dependence are modestly effective. Perhaps more importantly, it is becoming increasingly clear that these pharmacotherapies are more effective for some individuals than others. For example, preliminary work has suggested that naltrexone is more effective for individuals with a genetic variant that changes the binding profile of the $\mu$-opioid receptor (eg Oslin et al, 2003). These findings have led many to the conclusion that it is unlikely that we will have one pharmacotherapy that is effective for the majority of alcohol-dependent individuals. Instead, it is likely that a variety of pharmacotherapies will be developed, each of which may target different mechanisms, and, at the same

*Correspondence: Dr KE Hutchison, Department of Psychology, University of Colorado, Muenzinger Psychology Building D-244, Campus Box 345, Boulder, CO 80309-0345, USA, Tel: + I 303492 3298, Fax: + I 303492 2967, E-mail: kenth@psych.colorado.edu Received I4 February 2005; revised 2 August 2005; accepted 15 August 2005

Online publication: I September 2005 at http://www.acnp.org/citations/ Npp090 I05050096/default.pdf time, target individuals who are vulnerable on these specific mechanisms. Based on this notion, it is imperative to begin thinking about how best to test and develop treatments that target specific mechanisms that play an important role in the etiology of alcohol dependence and relapse. Likewise, it is equally important to describe individual differences in these mechanisms that are likely to predict the treatment outcome.

The action of alcohol and other drugs on the mesolimbic dopamine pathways is thought to be an important mechanism in the etiology of alcohol and drug dependence, and, more specifically, the development of intense craving and loss of control (Berridge and Robinson, 1998; Robinson and Berridge, 1993; Wise, 1988). While both the animal and human literature suggests that mesolimbic and prefrontal brain structures with dopaminergic connections are important, the role of specific dopamine receptor subtypes remains unclear. However, recent studies have indicated that the $\mathrm{D}_{2}$ family of dopamine receptors $\left(\mathrm{D}_{2}, \mathrm{D}_{3}\right.$, and $\left.\mathrm{D}_{4}\right)$ may play a critical role in incentive sensitization. For example, $\mathrm{D}_{4}$ receptors are localized to the same structures (eg orbitofrontal cortex, amygdala, and hippocampus) that have been implicated in both animal and human models of addiction and appetitive motivation (Oak et al, 2000; Asghari et al, 1995; Van Tol et al, 1991). In addition, selective $\mathrm{D}_{4}$ receptor antagonists block the sensitization of 
these dopamine pathways, suggesting that the $\mathrm{D}_{4}$ receptor is important to the process of sensitization (Feldpausch et al, 1998). $\mathrm{D}_{4}$ receptors that are localized in the shell of the nucleus accumbens may modulate excitatory transmission (Svingos et al, 2000). This research suggests that the $\mathrm{D}_{4}$ receptor may be central to the development of incentive sensitization and craving, and that the $D_{4}$ receptor may be generally involved in both the acquisition and expression of incentive salience.

With respect to clinical research, previous studies have indicated that craving for alcohol is diminished by dopamine antagonists (Modell et al, 1993), and that alcohol and alcohol cues increase activation in the same neuronal substrates that serve the process of incentive sensitization in humans (Modell and Mountz, 1995). Thus, the effect of alcohol and alcohol cues on the activation of these dopamine pathways represents a target that may prove to be useful in terms of pharmacotherapy development, and genetic variants that alter the functioning of these pathways may prove to be important in terms of predicting the success of such a pharmacotherapy. Other research has suggested that clozapine, a $\mathrm{D}_{4}$ receptor antagonist that is somewhat similar to olanzapine, reduced substance abuse among patients with comorbid substance abuse/dependence (Green et al, 1999) and, specifically, alcohol use (Drake et al, 2000). Thus, clinical research also supports an important role for the $\mathrm{D}_{2}$ family of receptors, and specifically the D4 receptor.

Given the potential importance of the dopamine pathways, and specifically the D4 receptor, a series of studies were conducted to examine whether a second-generation D2/D4 antagonist (eg olanzapine) reduced craving for alcohol and whether a functional variable number of tandem repeats (VNTR) polymorphism in the $\mathrm{D}_{4}$ receptor gene (DRD4) might moderate these effects. The $\mathrm{D}_{4}$ dopamine receptor gene (DRD4) has a VNTR polymorphism in exon 3, with common variants of two, four, and seven repeats (Van Tol et al, 1992). Previous work has suggested that the seven-repeat allele either alters intracellular function (Asghari et al, 1995; Oak et al, 2000) and/or suppresses expression by altering mRNA stability or translational efficiency (Schoots and Van Tol, 2003). The first study found that olanzapine reduced craving after exposure to the sight and smell of alcohol, as well as consumption of small doses of alcohol (Hutchison et al, 2001). A subsequent investigation found that the DRD4 VNTR polymorphism moderated the effects of alcohol on craving and other variables such that individuals with the seven-repeat allele demonstrated significantly higher craving after consumption of alcohol as compared to the control beverage, while individuals with the short-repeat alleles did not (Hutchison et al, 2002).

To replicate and extend the results with olanzapine and the DRD4 VNTR, a third study was designed to examine whether olanzapine $(5 \mathrm{mg})$ reduced craving as compared to cyproheptadine $(4 \mathrm{mg})$, which was conceptualized as an active control medication. Olanzapine is a potent $\mathrm{D}_{4}, \mathrm{D}_{2}, 5$ $\mathrm{HT}_{2}$, and $\mathrm{H}_{1}$ antagonist that may cause drowsiness, whereas cyproheptadine is also a powerful $5-\mathrm{HT}_{2}$ and $\mathrm{H}_{1}$ antagonist that may cause drowsiness. Thus, cyproheptadine represents an active control that can be used to better isolate the effect of olanzapine and exclude alternative explanations (eg drowsiness as a causal agent). It is important to note that there are very few published studies that have used such a stringent experimental control in a test of a pharmacological agent that targets alcohol craving. In this study, olanzapine was found to be much more effective at attenuating craving among individuals with the sevenrepeat allele (Hutchison et al, 2003).

None of the aforementioned studies examined the effect of olanzapine on drinking behavior in an alcohol-dependent population. A recent report suggested that olanzapine did not attenuate drinking in an alcohol-dependent population (Guardia et al, 2004). However, the investigators did not stratify subjects by the DRD4 genotype and they used doses of olanzapine that were two to three times greater than the doses shown to attenuate craving in our previous studies. The objective of the present study was to extend our previous results by testing the effects of olanzapine in an alcohol-dependent population over the course of a 12-week trial. More specifically, the present study utilized the dose of olanzapine that was supported in our previous studies (ie $5 \mathrm{mg}$ ) and stratified patients by DRD4 genotype. The effect of olanzapine on cue-elicited craving was assessed after 2 weeks of treatment, while the effect of olanzapine on drinking behavior was assessed after 4, 8, and 12 weeks of treatment. Consistent with our preliminary work, it was hypothesized that olanzapine would decrease cue-elicited craving and reduce drinking among the DRD4 L individuals.

\section{METHODS}

\section{Participants}

The present study was approved by the University of Colorado Human Research Committee, and all subjects provided written informed consent after receiving a full explanation of the study. Participants were recruited by newspaper or radio advertisements. All female subjects tested negative for pregnancy prior to participation, all subjects were required to have a blood alcohol concentration of zero before each session, and all subjects were required to be in excellent health, as indicated by a thorough medical screening designed to ensure that there were no contraindications for the use of the study medication. Subjects were also excluded if they met the criteria for specific psychiatric diagnoses (ie bipolar disorder, schizophrenia, bulimia, or anorexia nervosa), reported a psychological disorder requiring pharmacotherapy, endorsed current use of illicit drugs other than marijuana, or tested positive for the use of illicit drugs other than marijuana. Subjects were only included if they met DSM IV criteria for alcohol dependence. Furthermore, there was a minimum drinking requirement of 14 drinks (females) or 21 drinks (males) on average per week for four consecutive weeks. Participants also had to be within 21 days of their last drink to be included in the study.

Research participants were screened medically at the University of Colorado at Boulder General Clinical Research Center (GCRC). To be included in the trial, patients needed a normal medical exam, CBC, EKG, urine toxicology screen, and liver function tests that were within $3 \times$ the normal limit. A total of 154 subjects were assessed for eligibility, 78 
of whom met the full criteria for participation in the study. Of these 78,13 did not start the trial within 21 days of their last drink and were not included in the analyses.

\section{Medication Administration Procedures}

Prior to medication randomization, all participants were instructed to remain abstinent from alcohol for a minimum of 4 days. Participants were randomized to olanzapine or placebo. Participants were instructed to take $2.5 \mathrm{mg}$ of the study medication (either olanzapine or a matching placebo) for the first 5 days of treatment. On the sixth day, participants increased to $5 \mathrm{mg}$ per day dose for the remainder of the 12 weeks. Participants were also instructed to take the medication $3 \mathrm{~h}$ prior to going to bed in order to diminish the impact of any drowsiness experienced as a result of the olanzapine. The participants were instructed to report any side effects to the study physician or nurse at the GCRC. At the discretion of the medical staff at the GCRC, participants were instructed to decrease their dose back to $2.5 \mathrm{mg}$ to alleviate side effects or discontinue the study medication. Over the course of the study, eight participants in the olanzapine condition were reduced from the $5 \mathrm{mg}$ dose to the $2.5 \mathrm{mg}$ dose, while two participants in the placebo dose were reduced from 5 to $2.5 \mathrm{mg}$.

In order to confirm that participants took the medication, the medications were packed into an opaque capsule with $50 \mathrm{mg}$ of riboflavin. A urine sample was collected on the morning of the experimental session. The urine sample was tested for riboflavin content by examining it under an ultraviolet light, a procedure that makes the riboflavin detectable (Del Boca et al, 1996). None of the samples tested negative for riboflavin content.

\section{Experimental Procedures}

Individuals interested in the study called the laboratory and completed a telephone screening assessment. Eligible participants were then invited to a secondary screening visit, which consisted of a structured clinical interview (Structured Clinical Interview for DSM IV - SCID Clinician Version) assessing for alcohol dependence and concurrent psychiatric diagnoses. In addition to the clinical interview, participants completed the medical screening visit at the GCRC during this initial appointment. As stated above, the medical visit was designed to ensure that there were no contraindications to the use of study medications.

Eligible participants were invited back to the laboratory for a baseline session (session 1), during which participants completed measures of demographics, personality, alcohol use, alcohol-related problems, and motivation for change. At this time, participants also met with a therapist for the psychosocial and medication management component of this trial (described in detail below). At the end of session 1, participants were randomized to receive either olanzapine or placebo.

The cue reactivity session (session 2 ) took place 2 weeks after session 1. Following standardized procedures previously reported in the literature (eg Rohsenow et al, 2000), participants took part in a cue-exposure paradigm. In general, this procedure involves comparing reactivity between control cues (eg a nonalcoholic beverage) $v s$ an alcohol-related cue (eg the preferred alcoholic beverage). For the purposes of this study, Gatorade was used as the control cue, whereas the individuals' favorite alcoholic beverage served as the alcohol cue. During the control cue exposure, participants were asked to lift and sniff the Gatorade, but were not allowed to taste the Gatorade for a period of $3 \mathrm{~min}$. Participants completed measures of mood and craving (see below) before and after exposure to the control cues. After a 5-min relaxation period, participants repeated the cue exposure with their preferred alcohol beverage and subsequently completed the same measures of craving and mood. At the end of the cue exposure session, participants met with a therapist to process their reactivity to the cues, to discuss urge coping strategies, and to followup on their treatment goals.

Participants were also assessed for outcome and side effects during treatment on weeks 4,8 , and 12. Lastly, medication compliance was checked through a urine screen at the beginning of session 2 and at each assessment visit.

\section{Brief Psychosocial Intervention}

All participants receive two sessions of a brief structured psychosocial intervention. These sessions took place at the end of the baseline session (session 1) and the cue reactivity session (session 2). The sessions consisted of providing feedback on clients' drinking behavior (measured by study questionnaires), eliciting pros and cons about drinking alcohol, setting treatment goals, and eliciting strategies for achieving treatment goals. The therapists in the study were three female doctoral students in clinical psychology at the University of Colorado at Boulder. Individual therapists were crossed with treatment condition such that each therapist worked with equal numbers of participants in each of the medication conditions.

\section{Measures}

During the first experimental session, participants completed a battery of individual difference measures that included demographics, drinking behavior, personality dimensions, and motivation for change. Participants also completed a series of outcome measures, including medication side effects, starting 2 weeks after medication randomization. In addition, measures of urge to drink and affect were included to the cue-exposure paradigm, conducted during the second experimental session. The following measures were utilized in this study:

Stages of change readiness and treatment eagerness scale (SOCRATES). This is a 19-item measure of motivational processes associated with the stages of change model. Specifically, the SOCRATES consists of three subscales assessing recognition of alcohol-related problems (Recognition subscale), uncertainty about drinking (Ambivalence subscale), and taking action to change drinking behavior (Taking Steps subscale). The SOCRATES has been shown to be a valid and reliable measure of motivation for change (Miller and Tonigan, 1996).

A 30-day time-line follow-back procedure (TLFB; Sobell and Sobell, 1980). This measure was used to assess the 
quantity and frequency of drinking in the 30 days prior to the experiment. Consistent with previous outcome studies (eg Project MATCH Research Group, 1998; Monti et al, 2001; Killeen et al, 2004), the primary outcome variables were drinks per drinking day, total number of drinks, and percent days abstinent.

Years of sustained drinking. This variable was assessed by the item, 'How many years have you been drinking the quantity of alcohol per week that you are drinking now?'

Side-effect checklist. The short form of the Systematic Assessment for Treatment Emergent Events (SAFTEE) was utilized at each time point in the trial. This measure is recommended for use in clinical trials (Levine and Schooler, 1986; Jacobson et al, 1986) and consists of a survey of 24 common drug side effects (scored as present or absent).

Check on blind. Participants indicated which of the medications that they believed they received at the beginning of the cue-reactivity session.

Alcohol urge questionnaire (AUQ). The AUQ was used to assess urge to drink. The AUQ consists of eight items related to urge to drink that are rated on a 7-point Likert scale, with the extremes anchored by 'Strongly Disagree' and 'Strong Agree'. The AUQ has demonstrated high reliability (Bohn et al, 1995).

Profile of mood states (POMS). The POMS is a 40-item questionnaire assessing for multiple mood dimensions (McNair et al, 1971). The POMS has been used extensively in laboratory studies of the effects of alcohol and was utilized in this study to measure changes in mood during the cue-exposure paradigm.

\section{DNA Extraction and Genetic Analysis}

Genomic DNA was isolated from buccal cells using published procedures (Lench et al, 1988). The 48-bp VNTR in the third exon of the DRD4 was assayed using previously reported methods (Sander et al, 1997). The primer sequences were forward, $5^{\prime}$-AGGACCCTCATGGCCTTG- $3^{\prime}$ (fluorescently labeled), and reverse, 5'-GCGACTACG TGGTCTACTCG-3' (Lichter et al, 1993). PCR conditions were as follows: $200 \mu \mathrm{M}$ of each of the four dNTPs, $2.5 \mathrm{mM}$ $\mathrm{MgCl}_{2}, 200 \mathrm{nM}$ forward primer (fluorescently labeled), $200 \mathrm{nM}$ reverse primer, $40 \mathrm{ng}$ of genomic DNA, $1 \mathrm{U}$ of AmpliTaq Gold polymerase (ABI), and $1 \times$ PCR II buffer $(\mathrm{ABI})$ in a total volume of $20 \mu \mathrm{l}$. Amplification was performed with the following conditions: $95^{\circ} \mathrm{C}$ for $10 \mathrm{~min}$ (to activate the Gold polymerase); 35 cycles of $94^{\circ} \mathrm{C}$ for $30 \mathrm{~s}$, $55^{\circ} \mathrm{C}$ for $30 \mathrm{~s}$, and $72^{\circ} \mathrm{C}$ for $60 \mathrm{~s}$; and a final elongation at $72^{\circ} \mathrm{C}$ for $30 \mathrm{~min}$. PCR products were electrophoresed in $4.25 \%$ polyacrylamide under denaturing conditions with Applied Biosystems 3100. Participants were classified as DRD4 L (ie homozygous or heterozygous for an allele $\geqslant 7$ repeats; S/L or L/L), or were classified as DRD4 S (ie both alleles $<7$ repeats; $S / S$ ). Table 1 provides the allele and genotype frequencies.
Table I DRD4 VNTR Allele and Genotype Frequencies

\begin{tabular}{|c|c|c|}
\hline Allele/genotype & $n$ & $\%$ \\
\hline \multicolumn{3}{|l|}{ Allele } \\
\hline 2 & 10 & 8 \\
\hline 3 & 8 & 6 \\
\hline 4 & 80 & 63 \\
\hline 5 & । & 0.5 \\
\hline 7 & 27 & 21 \\
\hline 8 & 2 & 1.5 \\
\hline Total & 128 & \\
\hline \multicolumn{3}{|l|}{ Genotypes } \\
\hline $2 / 2$ & I & 1.5 \\
\hline $2 / 3$ & 2 & 3 \\
\hline $2 / 4$ & 5 & 8 \\
\hline $2 / 7$ & I & 1.5 \\
\hline $3 / 4$ & 4 & 6 \\
\hline $3 / 7$ & I & 1.5 \\
\hline $4 / 4$ & 30 & 47 \\
\hline $4 / 5$ & । & 1.5 \\
\hline $4 / 7$ & 12 & 19 \\
\hline $7 / 7$ & 6 & 9 \\
\hline $7 / 8$ & I & 1.5 \\
\hline Total & 64 & \\
\hline \multicolumn{3}{|c|}{ Genotype classification } \\
\hline$L$ & 22 & 34 \\
\hline $\mathrm{S}$ & 44 & 66 \\
\hline Total & 64 & \\
\hline
\end{tabular}

\section{RESULTS}

\section{Overview}

A series of analyses were conducted to determine whether the medication $\times$ DRD4 groups differed on drinking and demographic variables (see Table 2 for the means and standard deviations for the medication $\times$ DRD4 groups). Analyses of the baseline data suggested that the groups did not differ on these variables including ethnic background. Thus, it is highly unlikely that population stratification, gender, or other variables measured at baseline confounded the statistical analyses.

\section{Cue-Elicited Craving and Drinking Behavior}

Analyses were then conducted to determine whether the groups differed in terms of cue-elicited craving after 2 weeks of treatment and in terms of drinking behavior over the 12-week trial. These analyses utilized an 'intent-to-treat' approach such that all individuals who returned for the cue reactivity assessment at 2 weeks were included in the analyses, even if they dropped out of the trial. Six individuals in the olanzapine group and seven in the 
Table 2 Pretest Differences between the Medication Groups

\begin{tabular}{|c|c|c|c|c|}
\hline \multirow[b]{2}{*}{ Variable $^{\mathrm{a}}$} & \multicolumn{2}{|c|}{ Olanzapine } & \multicolumn{2}{|c|}{ Placebo } \\
\hline & DRD4 S $(n=23)$ & DRD4 L $(n=10)$ & DRD4 S $(n=19)$ & DRD4 L $(n=12)$ \\
\hline Ethnicity (\% caucasian) & $91 \%$ & $70 \%$ & $95 \%$ & $67 \%$ \\
\hline Age & $44.4(7.5)$ & $44.9(7.0)$ & $42.8(7.7)$ & $45.4(4.8)$ \\
\hline Years of sustained drinking & $23.1(9.1)$ & $20(9.2)$ & $20.6(7.8)$ & $23(5.2)$ \\
\hline 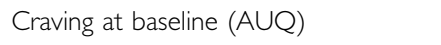 & $2.2(1.2)$ & $2.2(1.4)$ & $2.3(1.2)$ & $2.9(1.6)$ \\
\hline Taking steps to quit (SOCRATES) & $3.9(0.8)$ & $4.2(0.8)$ & $3.9(0.9)$ & $3.9(0.9)$ \\
\hline Recognition of problem (SOCRATES) & $4.1(0.6)$ & $4.1(0.8)$ & $3.9(0.9)$ & $4.3(0.7)$ \\
\hline Ambivalence (SOCRATES) & $4.2(0.7)$ & $4.1(0.6)$ & $3.8(0.9)$ & $3.7(1.1)$ \\
\hline
\end{tabular}

${ }^{a}$ Standard deviations appear in parentheses below the means of continuous variables.

placebo group dropped out of the study after the cue reactivity assessment, and were included in the analyses. For these individuals the last observation was carried forward in the analyses, following an intent-to-treat approach.

To analyze reactivity to alcohol cues, a series of $2 \times 2 \times 2$ mixed design analyses of variance (ANOVAs) were conducted, where cue (control cues $v s$ alcohol cues) was a two-level within-subjects factor, medication (olanzapine or placebo) was a two-level between-subjects factor, and DRD4 (DRD4 L vs DRD4 S) was a two-level between-subjects factor. The craving score at baseline was used as a covariate to control for the nonspecific effects of olanzapine on craving.

The analyses revealed a significant medication $\times$ DRD $4 \times$ cue interaction, $\mathrm{F}(1,59)=6.14, p<0.05$, and a significant DRD4 $\times$ cue interaction, $\mathrm{F}(1,59)=10.29, p<0.01$. Cue-elicited craving was clearly greater among DRD4 L individuals and olanzapine attenuated craving specifically among these individuals (see Figure 1). There was no significant main effect of olanzapine on craving $(p>0.05)$. With respect to changes in negative affect, the analyses revealed a significant medication $\times$ DRD $4 \times$ cue interaction, $\mathrm{F}(1,59)=8.11, \quad p<0.01$, a significant medication $\times$ cue interaction, $\mathrm{F}(1,59)=5.58, p<0.05$, and a significant main effect for cue, $F(1,59)=8.73, p<0.01$. Likewise, analyses revealed a significant medication $\times$ DRD $4 \times$ cue interaction on the Tension scale, $\mathrm{F}(1,59)=6.88, p<0.05$, as well as a significant main effect for cue and $F(1,59)=7.91$, $p<0.01$. These results suggested that olanzapine attenuated cue-induced increases in depression and anxiety, particularly among the DRD4 L individuals (see Figures 2 and 3 ).

To analyze the effects of olanzapine on drinking behavior, a series of $2 \times 2 \times 2$ mixed design ANOVAs were conducted, where medication (olanzapine vs placebo) was a two-level between-subjects factor, DRD4 (DRD4 L vs DRD4 S) was a two-level between-subjects factor, and Assessment (baseline, 4,8 , and 12 weeks) was a four-level within-subjects factor. Analyses revealed a significant medication $\times$
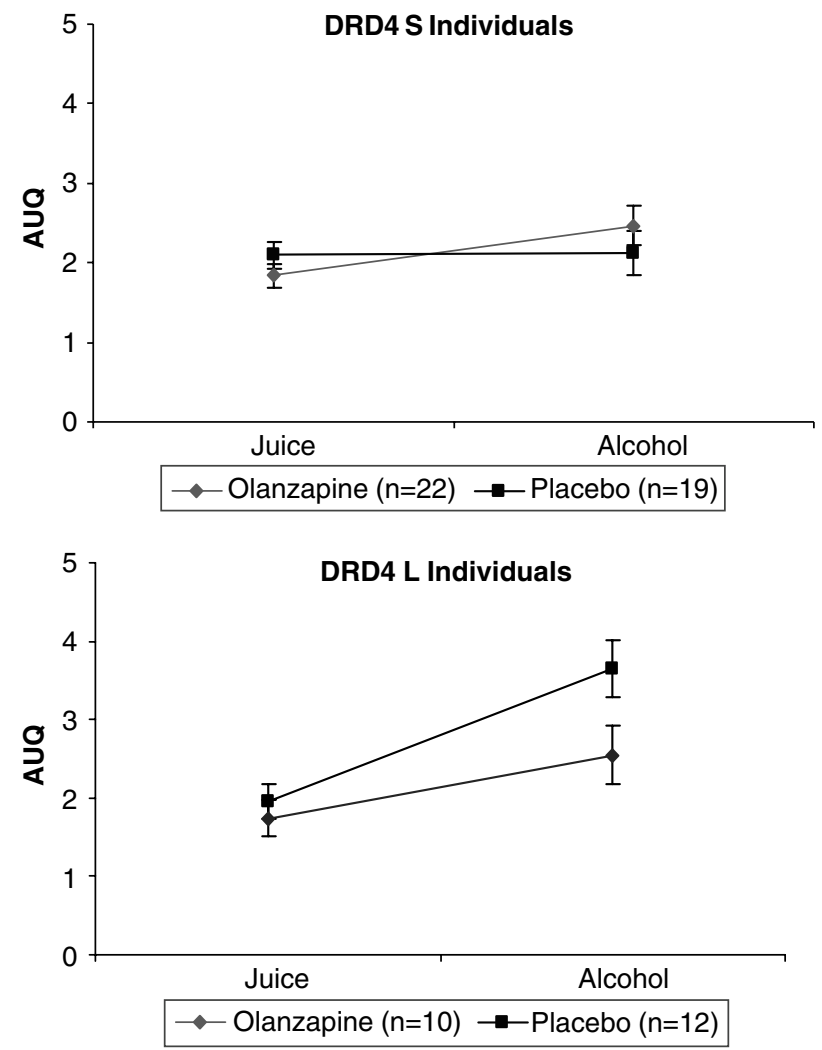

Figure I Mean craving (AUQ) scores at baseline and after exposure to alcohol cues. Analyses indicated a medication $\times$ DRD $4 \times$ trial interaction $(p<0.05)$ such that olanzapine significantly reduced craving at baseline and after cue exposure among the DRD4 $\mathrm{L}$ individuals and reduced craving at baseline among the DRD4 S individuals. Olanzapine did not reduce cueelicited craving among the DRD4 S individuals.

DRD $4 \times$ Assessment interaction on drinks per drinking day, $\mathrm{F}(3,180)=3.02, p<0.05$, and a significant main effect for medication, $\mathrm{F}(1,60)=4.88, p<0.05$. Olanzapine significantly reduced drinks per drinking day among the DRD4 L individuals (see Figure 4). An analysis of total number of 

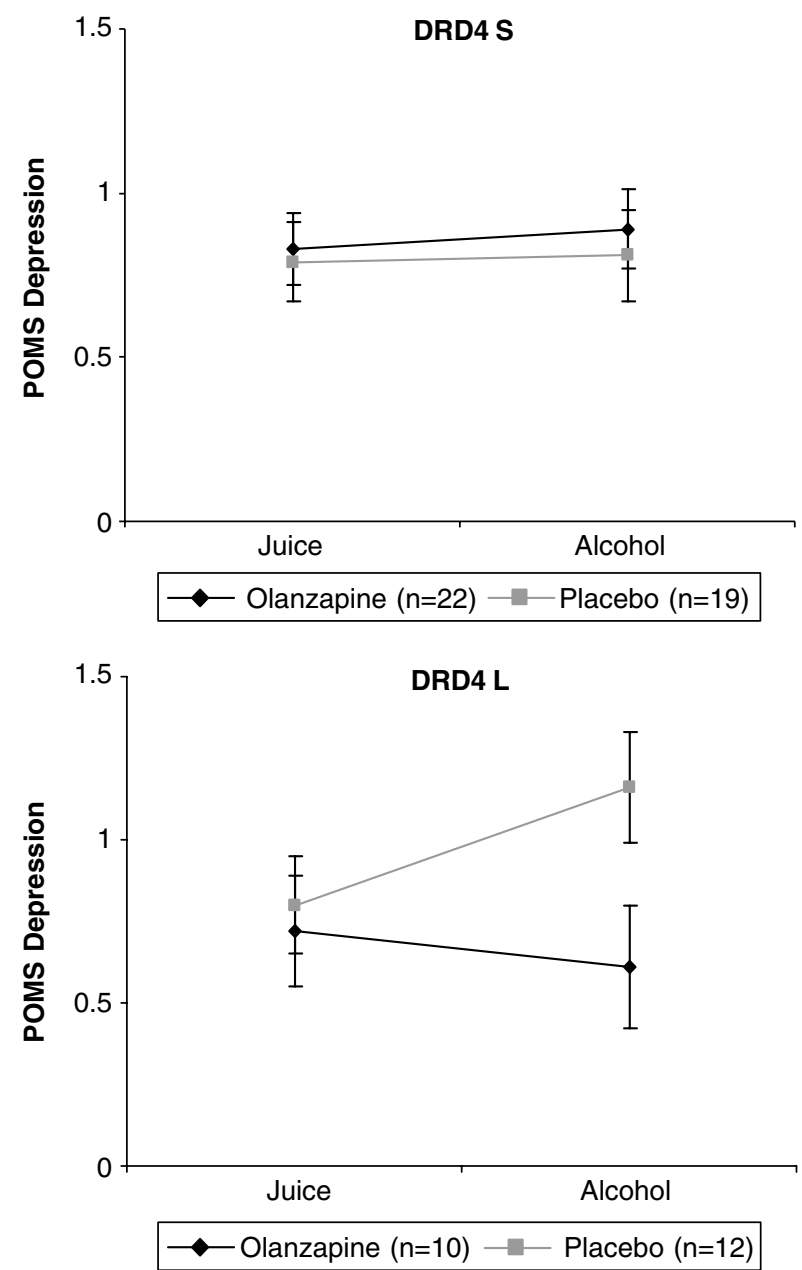

Figure 2 Mean score on the Depression subscale of the POMS and standard errors before and after cue exposure to alcohol for the DRD4 S group and the DRD4 L group. Analyses indicated that olanzapine significantly reduced the negative affect during the cue-reactivity paradigm among DRD4 $\mathrm{L}$ individuals, but had no effect on DRD4 S participants $(p<0.05)$

drinks also revealed a significant three-way interaction, $\mathrm{F}(3,180)=5.39, p<0.05$, indicating that olanzapine significantly reduced the total number of drinks for DRD4 L individuals. Similarly, an analysis of percent days abstinent revealed a trend for a medication $\times \mathrm{DRD} 4$ interaction, such that DRD4 $\mathrm{L}$ individuals responded more favorably to olanzapine $(p=0.06)$.

\section{Side Effects and Compliance}

Finally, analyses were conducted to assess whether side effects differed across medication groups. ANOVAs were used to examine change in weight, glucose levels, and GGT levels before and after the 12-week trial. With respect to weight gain, a significant medication $\times$ Time interaction indicated significant weight gain among individuals treated with olanzapine as compared to placebo, $\mathrm{F}(1,41)=6.54$, $p<0.01$. The average weight gain in the olanzapine group was $6.5 \mathrm{lbs}$ at the end of the 12 -week trial. There were no effects for the DRD4 on weight gain. Analyses of the blood
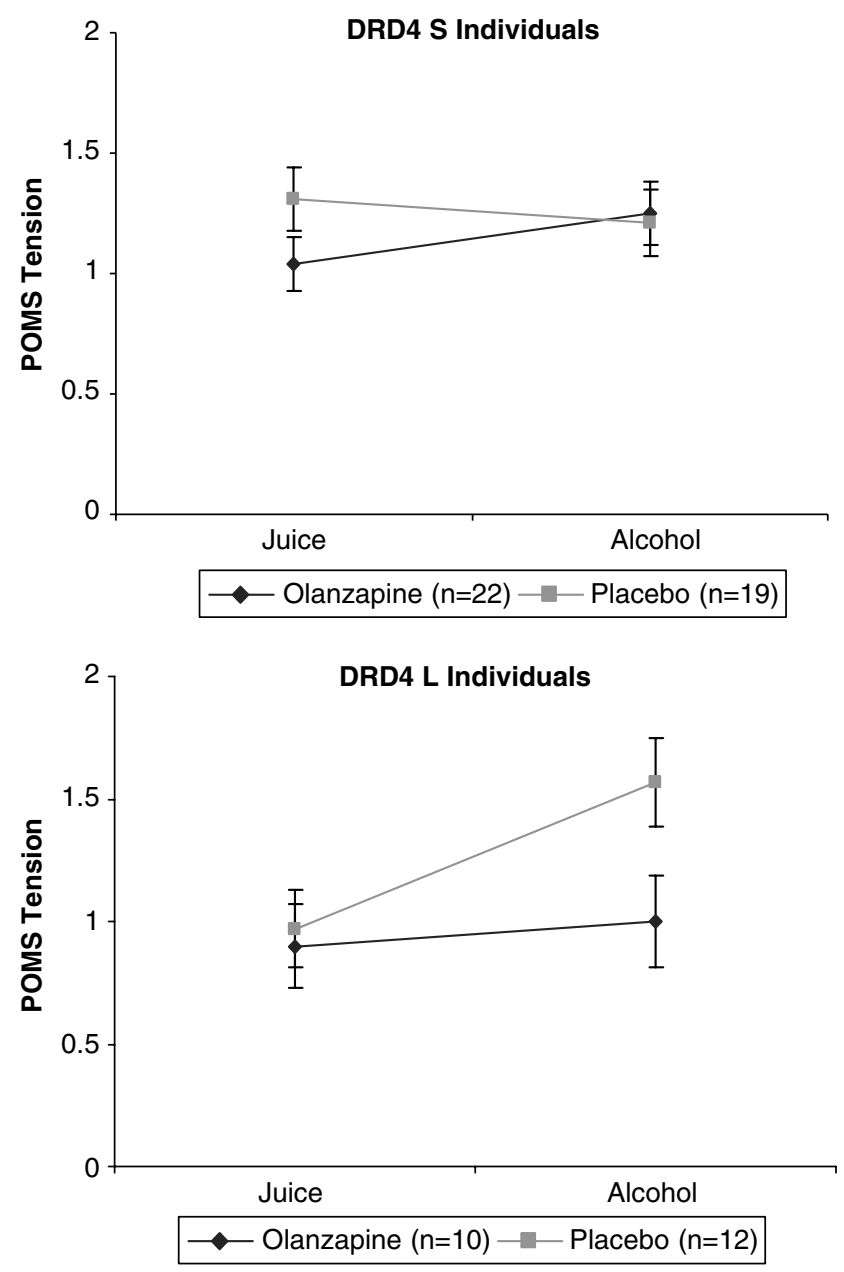

Figure 3 Mean score on the Tension subscale of the POMS and standard errors before and after cue exposure to alcohol for the DRD4 S group and the DRD4 L group. Analyses indicated that olanzapine significantly reduced tension during the cue-reactivity paradigm among DRD4 $\mathrm{L}$ individuals, but had no effect on DRD4 S participants $(p<0.01)$

glucose and GGT data revealed nonsignificant decreases across the 12-week trial, indicating that olanzapine did not have any deleterious effects in terms of blood glucose levels or liver function in this sample. Finally, $\chi^{2}$ tests were conducted comparing the medication groups on each of 24 items from the side effect checklist. None of these tests were significant $(p>0.10)$.

All urine samples tested positive for riboflavin, suggesting that individuals were compliant with the medication instructions immediately prior to each appointment. In addition, analyses of the pill count data suggested high compliance and did not reveal any significant differences in compliance across the medication $\times$ DRD4 groups. With respect to the integrity of the medication blind, $63 \%$ of the participants in the olanzapine condition guessed correctly at the beginning of the experimental session and $65 \%$ of the participants in the placebo condition guessed correctly. Finally, study completion status (completed $v s$ drop-out) and the integrity of the blind (guess correctly $v s$ did not guess correctly) were entered as covariates in the analyses 

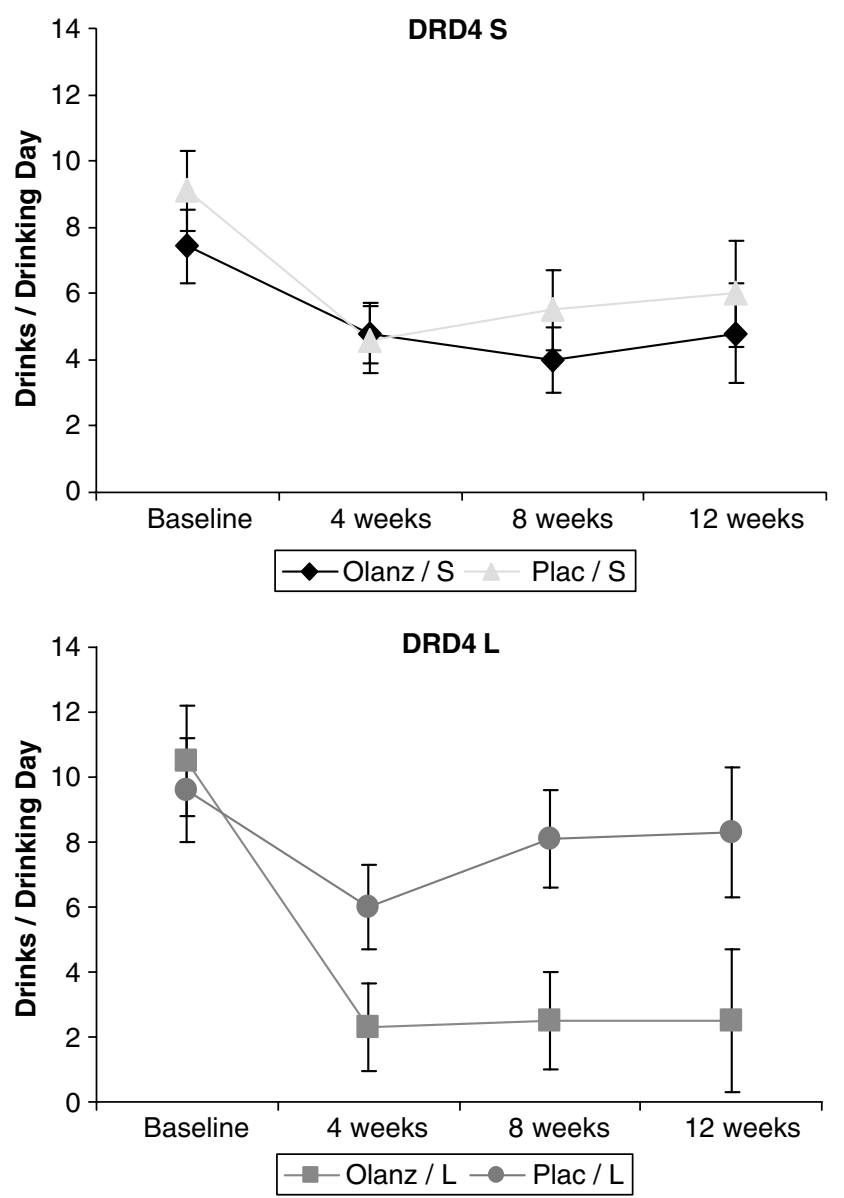

Figure 4 Mean number of drinks per drinking occasion and standard errors for the DRD L group and the DRD4 $S$ group across time points in trial. Analyses revealed a significant genotype $\times$ medication $\times$ trial interaction such that DRD4 L participants reported greater decreases in alcohol consumption across levels of trial as compared to DRD4 S individuals $(p<0.05)$.

described above. The significance of the results did not change, suggesting that neither of these variables biased the analyses.

\section{DISCUSSION}

The findings of the present study indicate that olanzapine reduces cue-elicited craving for alcohol and cue-elicited changes in affect among DRD4 L individuals after 2 weeks of treatment. Likewise, olanzapine appears to reduce the quantity of drinking over the course of a 12-week trial among DRD4 L individuals. The biological mechanism that underlies the differences in response to olanzapine among DRD4 $\mathrm{L}$ and S individuals is not clear. It is possible that the size of the intracytoplasmic loop may alter G protein coupling, which in turn may alter the function of the receptor. Future research will need to explore this question. With respect to side effects, individuals treated with olanzapine gained approximately $6.5 \mathrm{lbs}$ over the course of the 12-week study, regardless of their DRD4 genotype. However, there were no other statistically significant group differences with respect to side effects.
In the present study, it is important to note that $5 \mathrm{mg}$ of olanzapine did not appear to have any beneficial effects among the DRD4 S individuals. The lack of any significant effects of olanzapine among these individuals is consistent with a recent report that failed to find any beneficial effects of olanzapine during the course of a 12-week trial of olanzapine in an alcohol-dependent sample (Guardia $e t$ al, 2004). Conversely, the finding that olanzapine appears to attenuate reactivity to alcohol cues as well as drinking in DRD4 L individuals is not consistent with this report. There are two important differences between the present study and the previous report, which might explain the different findings. One of the primary differences is that the study by Guardia et al (2004) did not report results by DRD4 genotype, whereas genotype was considered in the present study. Second, a $10-15 \mathrm{mg}$ dose of olanzapine was used in the Guardia et al study, while $2.5-5 \mathrm{mg}$ was used in the present study. Previous work in our laboratory found that the $5 \mathrm{mg}$ dose of olanzapine attenuated craving for alcohol. Thus, the difference in findings may be explained in part by dose response differences and/or dose response by genotype differences.

The idea that higher doses of olanzapine may be associated with iatrogenic results is not surprising given that these doses are likely to produce more severe side effects, which in turn may lead to reduced compliance and/ or increased drinking as a means of coping with the side effects. While a dose of $10-20 \mathrm{mg}$ may be beneficial for individuals suffering from psychosis, these doses may not be appropriate for other disorders such as alcohol dependence. Even at $2.5-5 \mathrm{mg}$, olanzapine-treated individuals experienced significant weight gain in the present study, and, although other side effects observed in the olanzapine group were not significantly different from the side effects observed in the placebo group, a greater percentage of individuals in the olanzapine condition reported drowsiness. Clearly, these side effects may be more pronounced in a study using higher doses of olanzapine. In addition, olanzapine targets multiple neurotransmitter systems and the exact mechanism of its effect on craving and alcohol consumption is not known, although the DRD $4 \times$ olanzapine interaction is consistent with the hypothesis that the effect of olanzapine on the dopamine system is critical. Finally, it is possible that the DRD4 VNTR may shift the dose-response curve such that DRD4 S individuals might respond favorably to a different dose of olanzapine (eg a lower dose).

Given the potential importance of dosing and the possibility of a DRD $4 \times$ dose interaction, one of the limitations of the present study was the lack of a formal examination of the dose-response curve. Higher doses may produce no positive effects or even iatrogenic effects, while lower doses may be beneficial. Future studies should examine a $2.5 \mathrm{mg}$ dose as well as a $5 \mathrm{mg}$ dose, and stratify by genotype such that the hypothesis of a dose $\times$ genotype interaction can be formally tested. It seems possible that DRD4 $\mathrm{S}$ individuals may respond to $2.5 \mathrm{mg}$ even if they do not respond to $5 \mathrm{mg}$. To determine whether olanzapine will ultimately be useful in the treatment of alcohol dependence, future studies also need to examine the long-term effects of olanzapine on drinking behavior after treatment has ended. 


\section{ACKNOWLEDGEMENTS}

This research was supported by grants from the National Institute on Alcoholism and Alcohol Abuse (R01AA11473), the General Clinical Research Center Program of the National Center for Research Resources, National Institutes of Health (M01-RR00051), and a grant from Eli Lilly and Company.

\section{REFERENCES}

Asghari V, Sanyal S, Buchwaldt S, Paterson A, Jovanovic V, Van Tol HH (1995). Modulation of intracellular cyclic AMP levels by different human dopamine D4 receptor variants. J Neurochem 65: 1157-1165.

Berridge KC, Robinson TE (1998). What is the role of dopamine in reward: hedonic impact, reward learning, or incentive salience? Brain Res Brain Res Rev 28: 309-369.

Bohn MJ, Krahn DD, Staehler BA (1995). Development and initial validation of a measure of drinking urges in abstinent alcoholics. Alcohol Clin Exp Res 19: 600-606.

Del Boca FK, Kranzler HR, Brown J, Korner PF (1996). Assessment of medication compliance in alcoholics through UV light detection of a riboflavin tracer. Alcohol Clin Exp Res 20: 1412-1417.

Drake RE, Xie H, McHugo GJ, Green AI (2000). The effects of clozapine on alcohol and drug use disorders among patients with schizophrenia. Schizophr Bull 26: 441-449.

Feldpausch DL, Needham LM, Stone MP, Althaus JS, Yamamoto BK, Svensson KA et al (1998). The role of dopamine D4 receptor in the induction of behavioral sensitization to amphetamine and accompanying biochemical and molecular adaptations. J Pharmacol Exp Therap 286: 497-508.

Green AI, Zimmet SV, Strous RD, Schildkraut JJ (1999). Clozapine for comorbid substance use disorder and schizophrenia: Do patients with schizophrenia have a reward-deficiency syndrome that can be ameliorated by clozapine? Harvard Rev Psychiatry 6: $287-296$.

Guardia J, Segura L, Gonzalvo B, Iglesias L, Roncero C, Cardus M et al (2004). A double-blind, placebo-controlled study of olanzapine in the treatment of alcohol-dependence disorder. Alcohol Clin Exp Res 28: 736-745.

Hutchison KE, McGeary J, Smolen A, Bryan AD, Swift RM (2002). The DRD4 VNTR polymorphism moderates craving after alcohol consumption. Health Psychol 21: 139-146.

Hutchison KE, McGeary J, Smolen A, Wooden A (2001). Craving after alcohol consumption: olanzapine and the DRD4 VNTR polymorphism. Alcohol Clin Exp Res 25: 66a.

Hutchison KE, Wooden A, Swift RM, Smolen A, McGeary J, Adler L et al (2003). Olanzapine reduces craving for alcohol: a DRD4 VNTR polymorphism by pharmacotherapy interaction. Neuropsychopharmacology 28: 1882-1888.

Jacobson AF, Goldstein BJ, Dominguez RA, Steinbook RM (1986). Interrater agreement and intraclass reliability measures of SAFTEE in psychopharmacological clinical trials. Psychopharmacol Bull 22: 382-388.

Killeen TK, Brady KT, Gold PB, Tyson C, Simpson KN (2004). Comparison of self-report versus agency records of service utilization in a community sample of individuals with alcohol use disorders. Drug Alcohol Depend 73: 141-147.

Lench N, Stanier P, Williamson R (1988). Simple non-invasive method to obtain DNA for gene analysis. Lancet 1: 1356-1358.
Levine J, Schooler NR (1986). SAFTEE: a technique for the systematic assessment of side effects in clinical trials. Psychopharmacol Bull 22: 343-381.

Lichter JB, Barr CL, Kennedy JL, Van Tol HH, Kidd KK, Livak KJ (1993). A hypervariable segment in the human dopamine receptor D4 (DRD4) gene. Hum Mol Genet 2: 767-773.

McNair DM, Lorr M, Droppleman LF (1971). Profile of Mood States (Manual). Educational and Industrial Testing Service: San Diego, CA.

Miller WR, Tonigan JS (1996). Assessing drinkers' motivations for change: The Stages of Change Readiness and Treatment Eagerness Scale (SOCRATES). Psychol Addict Behav 10: 81-89.

Modell JG, Mountz JM (1995). Focal cerebral blood flow change during craving for alcohol measured by SPECT. J Neuropsychiatry Clin Neurosci 7: 15-22.

Modell JG, Mountz JM, Glaser FB, Lee JY (1993). Effect of haloperidol on measures of craving and impaired control in alcoholic subjects. Alcohol Clin Exp Res 17: 234-240.

Monti PM, Rohsenow DJ, Swift RM, Gulliver SB, Colby SM, Mueller TI et al (2001). Naltrexone and cue exposure with coping and communication skills training for alcoholics: treatment process and 1-year outcomes. Alcohol Clin Exp Res 25: 16341647.

Oak JN, Oldenhof J, Van Tol HH (2000). The dopamine D4 receptor: one decade of research. Eur J Pharmacol 405: 303-327.

Oslin DW, Berrettini W, Kranzler HR, Pettinati H, Gelernter J, Volpicelli JR et al (2003). A functional polymorphism of the opioid receptor gene is associated with naltrexone response in alcohol-dependent patients. Neuropsychopharmacology 28: 15461552.

Project MATCH Research Group (1998). Matching alcoholism treatments to client heterogeneity: Project MATCH three-year drinking outcomes. Alcohol Clin Exp Res 22: 1300-1311.

Robinson TE, Berridge KC (1993). The neural basis of drug craving: an incentive-sensitization theory of addiction. Brain Res Brain Res Rev 18: 247-291.

Rohsenow DJ, Monti PM, Hutchison KE, Swift RM, Colby SM, Kaplan GB (2000). Naltrexone's effects on reactivity to alcohol cues among alcoholic men. J Abnorm Psychol 109: 738-742.

Sander T, Harms H, Dufeu P, Kuhn S, Rommelspacher H, Schmidt LG (1997). Dopamine D4 receptor exon III alleles and variation of novelty seeking in alcoholics. Am J Med Genet 74: 483-487.

Schoots O, Van Tol HH (2003). The human dopamine D4 receptor repeat sequences modulate expression. Pharmacogenom $J$ 3: 343-348.

Sobell LC, Sobell MB (1980). Convergent validity: an approach to increasing confidence in treatment outcome conclusions with alcohol and drug abusers. In: Sobell LC, Sobell MB, Ward E (eds). Evaluating Alcohol and Drug Abuse Treatment Effectiveness: Recent Advances. Pergamon Press: New York. pp 177-183.

Svingos AL, Periasamy S, Pickel VM (2000). Presynaptic dopamine D4 receptor localization in the rat nucleus accumbens shell. Synapse 36: 222-232.

Van Tol HH, Bunzow JR, Guan H, Sunahara RK (1991). Cloning of the gene for a human dopamine D-sub-4 receptor with high affinity for the antipsychotic clozapine. Nature 350: 610-614.

Van Tol HH, Wu CM, Guan H, Ohara K (1992). Multiple dopamine D4 receptor variants in the human population. Nature 358: 149-152.

Wise RA (1988). The neurobiology of craving: implications for the understanding and treatment of addiction. J Abnorm Psychol 97: $118-132$. 